DRAFT VERSION APRIL 20, 2018

Preprint typeset using LTEX style emulateapj v. 5/2/11

\title{
FORMALDEHYDE SILHOUETTES AGAINST THE COSMIC MICROWAVE BACKGROUND: A MASS-LIMITED, DISTANCE-INDEPENDENT, EXTINCTION-FREE TRACER OF STAR FORMATION ACROSS THE EPOCH OF GALAXY EVOLUTION
}

\author{
JEREMY DARLING ${ }^{1,2,3}$ AND BENJAMIN ZEIGER ${ }^{1,4}$ \\ Draft version April 20, 2018
}

\begin{abstract}
We examine the absorption of cosmic microwave background (CMB) photons by formaldehyde $\left(\mathrm{H}_{2} \mathrm{CO}\right)$ over cosmic time. The $K$-doublet rotational transitions of $\mathrm{H}_{2} \mathrm{CO}$ become "refrigerated" - their excitation temperatures are driven below the CMB temperature - via collisional pumping by molecular hydrogen $\left(\mathrm{H}_{2}\right)$. "Antiinverted" $\mathrm{H}_{2} \mathrm{CO}$ line ratios thus provide an accurate measurement of the $\mathrm{H}_{2}$ density in molecular clouds. Using a radiative transfer model, we demonstrate that $\mathrm{H}_{2} \mathrm{CO}$ centimeter wavelength line excitation and detectability are nearly independent of redshift or gas kinetic temperature. Since the $\mathrm{H}_{2} \mathrm{CO} K$-doublet lines absorb CMB light, and since the CMB lies behind every galaxy and provides an exceptionally uniform extended illumination source, $\mathrm{H}_{2} \mathrm{CO}$ is a distance-independent, extinction-free molecular gas mass-limited tracer of dense gas in galaxies. A Formaldehyde Deep Field could map the history of cosmic star formation in a uniquely unbiased fashion and may be possible with large bandwidth wide-field radio interferometers whereby the silhouettes of star-forming galaxies would be detected across the epoch of galaxy evolution. We also examine the possibility that $\mathrm{H}_{2} \mathrm{CO}$ lines may provide a standardizable galaxy ruler for cosmology similar to the Sunyaev-Zel'dovich effect in galaxy clusters but applicable to much higher redshifts and larger samples. Finally, we explore how anti-inverted meterwave $\mathrm{H}_{2} \mathrm{CO}$ lines in galaxies during the peak of cosmic star formation may contaminate $\mathrm{H} \mathrm{I}$ $21 \mathrm{~cm}$ tomography of the Epoch of Reionization.
\end{abstract}

Subject headings: cosmic background radiation — dark ages, reionization, first stars — galaxies: high-redshift — galaxies: ISM — galaxies: star formation — radiation mechanisms: non-thermal

\section{INTRODUCTION}

Centimeter-wave $K$-doublet rotational transitions of formaldehyde $\left(\mathrm{H}_{2} \mathrm{CO}\right.$; Figure 1) can be collisionally "refrigerated" below the cosmic microwave background (CMB) temperature such that these transitions absorb CMB photons (Palmer et al. 1969; Townes \& Cheung 1969; Evans et al. 1975; Garrison et al. 1975). The manner in which the centimeter lines of $\mathrm{H}_{2} \mathrm{CO}$ are cooled is equivalent to a maser pumping process: a pump drives an over-population of states, but in this case they are lower-energy states, and the level populations become "anti-inverted" compared to thermal. When the line excitation temperature drops below the local CMB temperature, CMB photons may be absorbed. Since absorption lines are detectable independent of distance, and since the CMB illuminates all gas and provides an exceptionally uniform illumination that lies behind every galaxy, a Formaldehyde Deep Field (FDF) can provide a mass-limited census of the dense molecular gas associated with star formation. If multiple lines are observed then physical molecular gas densities may also be obtained fairly independently of the gas kinetic temperature or other factors (e.g., Mangum et al. 2008).

In this Letter, we examine the properties of the anti-inverted centimeter-wave $\mathrm{H}_{2} \mathrm{CO} \mathrm{K}$-doublet lines as a function of physical conditions and cosmological redshift in a feasibility study for a census of the cosmic gas evolution and star forma-

\footnotetext{
${ }^{1}$ Center for Astrophysics and Space Astronomy, Department of Astrophysical and Planetary Sciences, University of Colorado, 389 UCB, Boulder, CO 80309-0389

2 NASA Lunar Science Institute, NASA Ames Research Center, Moffett Field, CA

3 jdarling@ colorado.edu

${ }^{4}$ benjamin.zeiger@colorado.edu
}

tion history via an FDF. We also discuss the possibility that $\mathrm{H}_{2} \mathrm{CO}$ lines can provide a standardizable ruler for cosmology and may contaminate H I $21 \mathrm{~cm}$ tomography observations of the Epoch of Reionization (EoR). This work examines radiative transfer models only, but is supported by observations of our galaxy (Mangum \& Wootten 1993; Ginsburg et al. 2011), nearby starburst galaxies (Mangum et al. 2008), and the gravitational lens galaxy toward B0218+357 at $z=0.68$ (Zeiger \& Darling 2010).

We assume a no-curvature cosmology with $H_{\circ}=$ $72 \mathrm{~km} \mathrm{~s}^{-1} \mathrm{Mpc}^{-1}, \Omega_{m}=0.26$, and $\Omega_{\Lambda}=0.74$.

\section{RADIATIVE TRANSFER}

We use the RADEX one-dimensional non-LTE radiative transfer code with the escape probability determined by the large velocity gradient (LVG; Sobolev 1960; Goldreich \& Kwan 1974) approximation to examine the excitation and thus detectability and interpretation of $\mathrm{cm} \mathrm{H}_{2} \mathrm{CO}$ lines (Van der Tak et al. 2007). We employ the $\mathrm{H}_{2}$ collision rate coefficients calculated by Troscompt et al. (2009b) for the 10 lowest levels of ortho- $\mathrm{H}_{2} \mathrm{CO}$ (to $J=5$; Figure 1 ) for gas kinetic temperatures of 5-100 K. We have compared the results from this code with another LVG treatment (Henkel et al. 1980) and with RADEX (using the LVG approximation), both using the Green (1991) collision rates scaled from $\mathrm{He}$ to $\mathrm{H}_{2}$, and all are in good agreement for low $\mathrm{H}_{2}$ ortho-to-para ratio (OPR). The latter models include the first 40 energy levels of ortho- $\mathrm{H}_{2} \mathrm{CO}$; agreement between these models and those using only the 10 levels of Troscompt et al. (2009b) lends credibility to our treatment of warm molecular gas in star-forming galaxies.

For the radiative transfer calculations we assume statistical equilibrium but allow all line excitation temperatures to float 


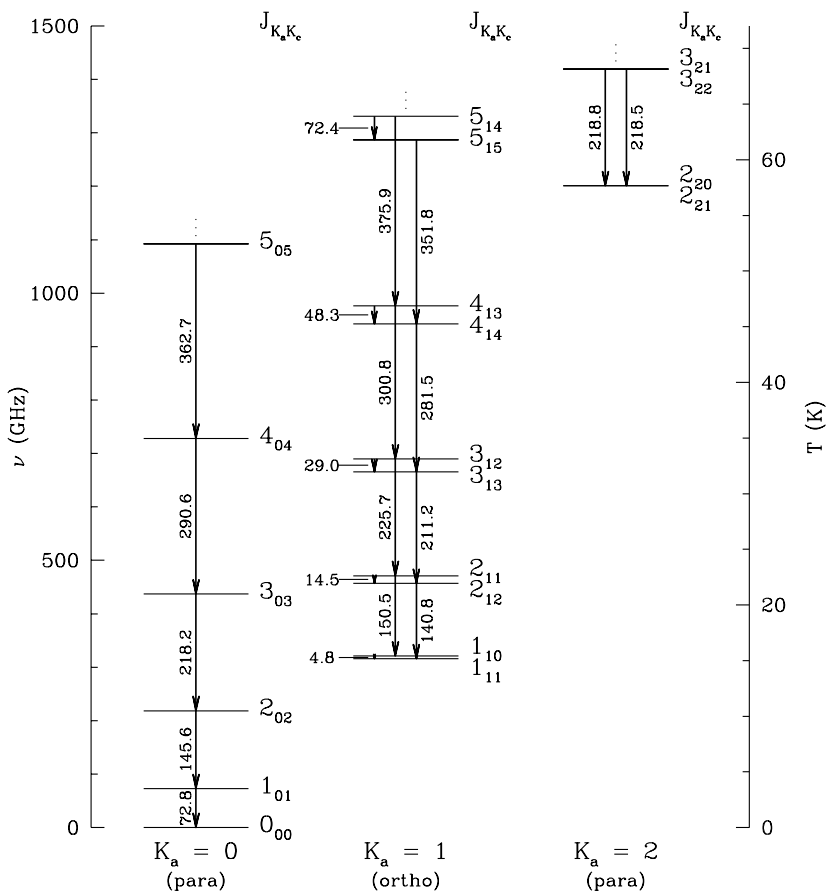

FIG. 1.- Formaldehyde energy levels for the first three rotation ladders. Arrows indicate allowed transitions, and line frequencies are listed in $\mathrm{GHz}$. The $K$-doublet levels splitting $J$ states of the $K_{a}=1$ ortho- $\mathrm{H}_{2} \mathrm{CO}$ rotation ladder are the focus of this Letter. The wavelengths of these transitions are, from lowest energy to highest, roughly $6,2,1,0.6$, and $0.4 \mathrm{~cm}$.

because the dominant collision partner, $\mathrm{H}_{2}$, "pumps" $\mathrm{H}_{2} \mathrm{CO}$ into non-thermal excitation state distributions. We assume that the CMB is the dominant continuum source and that there is no significant contribution to the radio continuum from the host galaxy itself. An examination of this effect in the starburst galaxy M81 shows a small effect that does not qualitatively alter our results (Mangum et al. 2008). We also assume an $\mathrm{H}_{2} \mathrm{CO} / \mathrm{H}_{2}$ abundance of $10^{-9}$, which is reasonable in molecular gas-rich galaxies, even at high redshift (Zeiger \& Darling 2010 obtain an abundance $0.1-2.5 \times 10^{-9}$ in the gravitational lens galaxy toward $\mathrm{B} 0218+357$ at $z=0.68$ ), and an $\mathrm{H}_{2} \mathrm{CO}$ OPR of 3 .

Troscompt et al. (2009a b) show that the anti-inversion of ortho- $\mathrm{H}_{2} \mathrm{CO}$ is principally driven by collisions with para- $\mathrm{H}_{2}$. Since the $\mathrm{H}_{2} \mathrm{CO}$ line excitation temperatures critically determine the detectability and interpretation of lines, the effect of the unknown $\mathrm{H}_{2}$ OPR is a concern. To address this, we examine model runs with $\mathrm{H}_{2}$ OPR of 3 and $10^{-2}$ (effectively zero) and find small differences in line excitation temperature $(\lesssim 0.3 \mathrm{~K})$ and no significant effect on the broad results and interpretation (Section 3). The basic phenomenon of antiinversion of $\mathrm{cm} \mathrm{H}_{2} \mathrm{CO}$ lines and their ability to produce silhouettes of star-forming galaxies against the CMB is robust to the unknown $\mathrm{H}_{2}$ OPR, as is the nearly temperature- and redshift-independent nature of the line detectability and excitation.

We examine the model predictions for $\mathrm{cm}_{2} \mathrm{CO}$ line excitation for varying $n\left(\mathrm{H}_{2}\right)$, gas kinetic temperature, and redshift. When not varying, we assume $n\left(\mathrm{H}_{2}\right)=10^{4} \mathrm{~cm}^{-3}, T_{\text {kin }}=40 \mathrm{~K}$, and $\mathrm{H}_{2}$ OPR $10^{-2}$ (e.g., Maret \& Bergin (2007), Pagani et al. (2009), and Troscompt et al. (2009a) infer ratios in the range 0.01-0.1 in cold Galactic pre-stellar molecular gas). We focus on the first five $K$-doublet lines because these produce the strongest signals (millimeter lines thermalize in typical

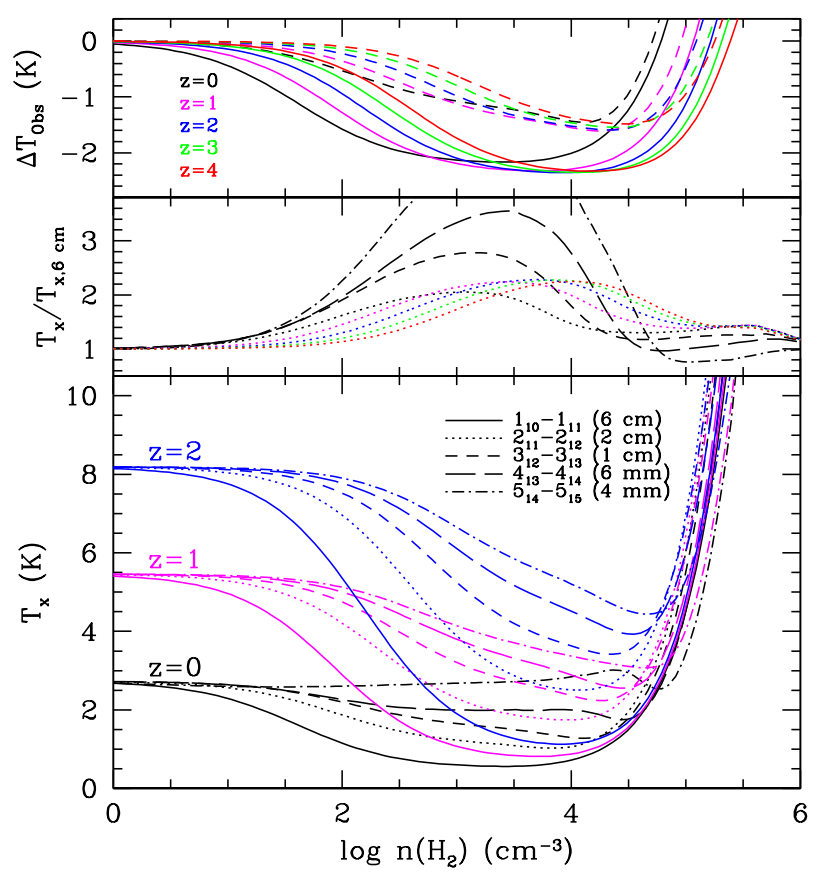

FIG. 2.- Formaldehyde line excitation vs. $\mathrm{H}_{2}$ number density. We plot several redshifts to demonstrate the excitation redshift dependence. Line style indicates transition, and color indicates redshift. Bottom: excitation temperature vs. $\log n\left(\mathrm{H}_{2}\right)$. Middle: excitation temperature normalized by the $6 \mathrm{~cm}$ line excitation temperature vs. $n\left(\mathrm{H}_{2}\right)$. We plot all five lines at $z=0$ and the $2 \mathrm{~cm}$ line at redshifts $z=0-4$, normalized to the $6 \mathrm{~cm}$ line at each redshift. Top: observer-frame temperature decrement, $\Delta T_{\text {Obs }}$ (Equation 1 for an optically thick line vs. $n\left(\mathrm{H}_{2}\right)$. We plot redshifts $z=0-4$ for the representative $6 \mathrm{~cm}$ and the $1 \mathrm{~cm}$ lines (other lines are omitted for clarity). $\Delta T_{\mathrm{Obs}}=0 \mathrm{~K}$ represents the transition from absorption to emission.

conditions), and the Troscompt et al. (2009b) calculations do not include higher states. These lines have wavelengths of roughly $6,2,1,0.6$, and $0.4 \mathrm{~cm}(4.8,14.5,29.0,48.3$, and $72.4 \mathrm{GHz}$, respectively).

The observed line temperature depends on the decrement between the rest-frame excitation temperature and CMB temperature, redshifted to $z=0$, and the line optical depth:

$$
\Delta T_{\mathrm{Obs}}=\frac{T_{\mathrm{x}}(z)-T_{\mathrm{CMB}}(z)}{1+z}\left(1-e^{-\tau}\right)
$$

where $T_{\mathrm{CMB}}(z)=T_{\mathrm{CMB}, 0}(1+z)$ and $T_{\mathrm{CMB}, 0}=2.73 \mathrm{~K}$. For a nonunity covering factor, the optical depth would be correspondingly diminished. Mangum et al. (2008) estimate the apparent line optical depths in nearby star-forming galaxies: 0.002 0.007 in the $6 \mathrm{~cm}$ line and $0.0015-0.005$ in the $2 \mathrm{~cm}$ line. Zeiger \& Darling (2010) estimate 0.017 at $6 \mathrm{~cm}$ and 0.008 at $2 \mathrm{~cm}$ in the molecular absorber toward B0218+357 at $z=0.67$. The Galactic range among ultracompact H II regions is similar, but with a higher upper bound: $0.006-1.0$ at $6 \mathrm{~cm}$ and $0.003-0.3$ at $2 \mathrm{~cm}$ (Ginsburg et al. 2011).

The goal of this work is to examine the observer-frame temperature decrement $\left(T_{\mathrm{x}}(z)-T_{\mathrm{CMB}}(z)\right) /(1+z)$ of $\mathrm{cm} \mathrm{H}_{2} \mathrm{CO}$ lines, which is equivalent to $\Delta T_{\mathrm{Obs}}$ for optically thick lines. We focus on the excitation temperature of the $\mathrm{cm} \mathrm{H}_{2} \mathrm{CO}$ lines as a function of gas kinetic temperature, $\mathrm{H}_{2}$ density, and redshift because these factors will most significantly determine the utility and interpretation of $\mathrm{H}_{2} \mathrm{CO}$ line observations at high redshift.

\section{RESULTS}




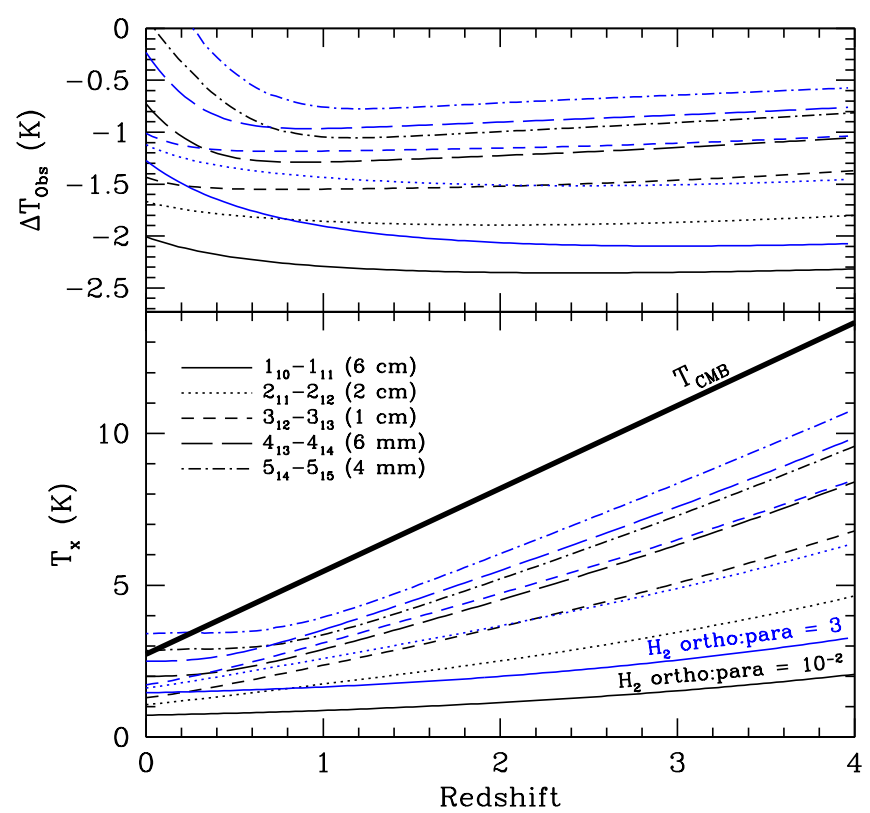

FIG. 3.- Formaldehyde line excitation vs. redshift. Line style indicates transition, and color indicates $\mathrm{H}_{2}$ OPR. Bottom: excitation temperature vs. redshift. The $\mathrm{CMB}$ temperature is bold. $T_{\mathrm{x}}<T_{\mathrm{CMB}}$ indicates absorption of CMB photons. Top: observer-frame temperature decrement $\Delta T_{\mathrm{Obs}}$ (Equation 11 for an optically thick line vs. redshift. The fairly flat trend in these decrements shows the nearly redshift-independent detectability of $\mathrm{cm} \mathrm{H}_{2} \mathrm{CO}$ lines.

Our radiative transfer models show that the excitation temperature of $\mathrm{cm} \mathrm{H}_{2} \mathrm{CO}$ lines is a strong function of $\mathrm{H}_{2}$ number density, collisionally driven below $T_{\mathrm{CMB}}$ over roughly three orders of magnitude, $10^{2} \mathrm{~cm}^{-3} \lesssim n\left(\mathrm{H}_{2}\right) \lesssim 10^{5} \mathrm{~cm}^{-3}$ (Figure 2). At low densities the $\mathrm{H}_{2} \mathrm{CO}$ transitions thermalize to the CMB temperature, $T_{\mathrm{x}} \rightarrow T_{\mathrm{CMB}}$, because the molecule becomes decoupled from the gas and assumes the radiation temperature. At high densities the $\mathrm{H}_{2} \mathrm{CO}$ excitation thermalizes to the kinetic temperature, $T_{\mathrm{x}} \rightarrow T_{\text {kin }}$.

The range over which $n\left(\mathrm{H}_{2}\right)$ causes anti-inversion of the $\mathrm{H}_{2} \mathrm{CO}$ lines shifts slightly to higher densities with increasing redshift, by $\sim 0.5$ dex from $z=0$ to $z=4$, but this is entirely due to the $T_{\mathrm{CMB}}=(1+z) 2.73 \mathrm{~K}$ scaling rather than a change in physical pumping conditions. The minimum $\Delta T_{\mathrm{Obs}}$ likewise grows slightly (becomes more negative) with increasing redshift, decreasing by $\sim 0.2 \mathrm{~K}$ from $z=0$ to $z \geq 1$.

For fixed $n\left(\mathrm{H}_{2}\right)$ and $T_{\text {kin }}, T_{\mathrm{x}}$ grows roughly linearly with redshift (Figure 3). But since the $\mathrm{CMB}$ temperature scales as $T_{\mathrm{CMB}}=(1+z) 2.73 \mathrm{~K}$, the redshift dependence largely drops out of $\Delta T_{\mathrm{Obs}}$, and the observable line decrement changes very weakly with redshift. Thus, not only is there no distance dimming of anti-inverted $\mathrm{cm} \mathrm{H}_{2} \mathrm{CO}$ lines thanks to the absorption of $\mathrm{CMB}$ photons, but the excitation conditions are not changed significantly by the growth of CMB temperature with redshift.

At each redshift, $T_{\text {kin }}$ has a $T_{\text {kin }}=T_{\mathrm{CMB}}=T_{\mathrm{x}}$ floor: the antiinversion disappears when the radiation, excitation, and gas kinetic temperatures are equal (Figure 4). The lower panel of Figure 4 indicates a nearly redshift-proportional line excitation: $T_{\mathrm{x}}$ becomes insensitive to $T_{\text {kin }}$ once collisions dominate the level populations, and redshift alone dictates $T_{\mathrm{x}}$. Except for $z \sim 0$, where $T_{\mathrm{CMB}}$ is very low, the line decrements are insensitive to $T_{\text {kin }}$ after an initial drop from $T_{\mathrm{x}}=T_{\mathrm{kin}}=T_{\mathrm{CMB}}$. In fact, higher energy $K$-doublet lines become anti-inverted at $z \gtrsim 1$. The 6 and $1 \mathrm{~cm}$ lines show excellent overlap in $\Delta T_{\mathrm{Obs}}$

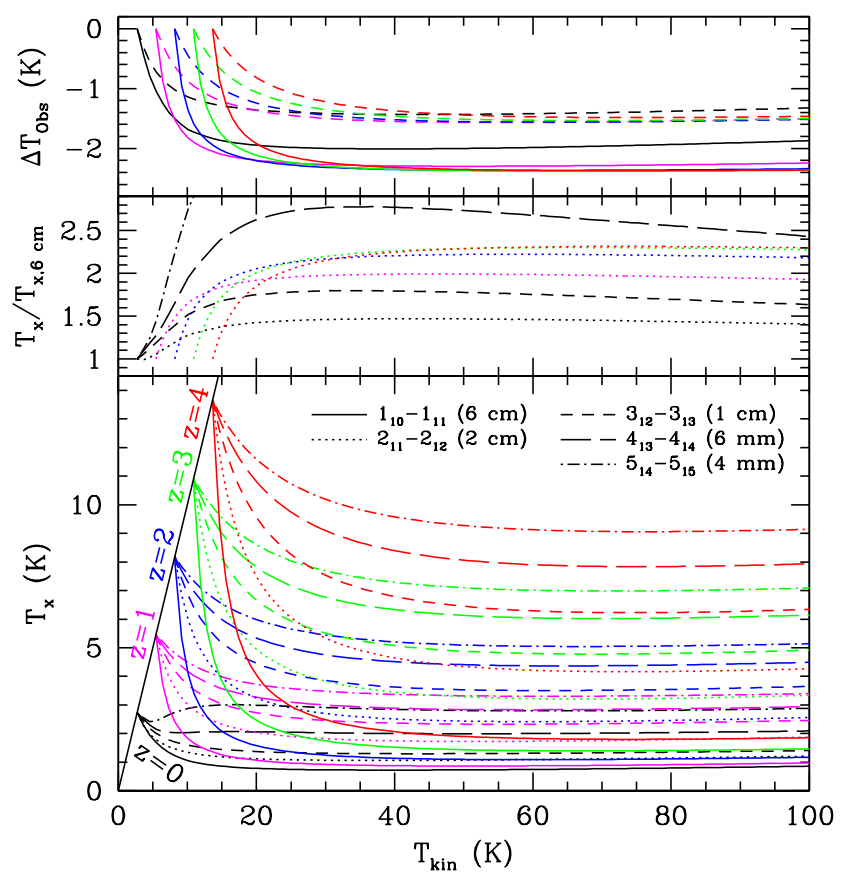

FIG. 4.- Formaldehyde line excitation versus gas kinetic temperature. We plot several redshifts to demonstrate the impact of the $(1+z)$ CMB temperature scaling. Line style indicates transition, and color indicates redshift. Bottom: excitation temperature versus $T_{\text {kin }}$. The black diagonal line shows $T_{\mathrm{X}}=T_{\mathrm{kin}}$, and the convergence point of the transitions at each redshift indicates where radiation, kinetic, and excitation temperatures are equal. Middle: excitation temperature normalized by the $6 \mathrm{~cm}$ line excitation temperature versus $T_{\text {kin. }}$. We plot all lines at $z=0$ and the $2 \mathrm{~cm}$ line at redshifts $z=0-4$ normalized to the $6 \mathrm{~cm}$ line at each redshift. Top: observer-frame temperature decrement $\Delta T_{\text {Obs }}$ (Equation 1 for an optically thick line versus $T_{\text {kin }}$. We plot redshifts $z=0-4$ for the representative $6 \mathrm{~cm}$ and the $1 \mathrm{~cm}$ lines (other lines omitted for clarity). After the plunge from $T_{\mathrm{kin}}=T_{\mathrm{x}}=T_{\mathrm{CMB}}, T_{\mathrm{X}}$ and $\Delta T_{\mathrm{Obs}}$ depend only weakly on $T_{\text {kin }}$ with the notable exception of the higher energy $z=0$ lines, which lie on the threshold of anti-inversion.

for $z \geq 1$ and a nearly $T_{\text {kin }}$-independent trend.

As the upper panel of Figure 4 shows, there is little change in the observed line depth versus redshift or $T_{\text {kin }}$ with the notable exception of the $6 \mathrm{~cm}$ line at $z \simeq 0$, and there is rapid convergence of all lines at $z \gtrsim 1$ for realistic values of $T_{\text {kin }}$ $(\gtrsim 20 \mathrm{~K})$. Figure 4 also demonstrates that there is little variation in the excitation temperature ratios between lines for $z \gtrsim 1$ as a function of gas kinetic temperature. Since line ratios indicate the $\mathrm{H}_{2}$ molecular gas density, this demonstrates that we do not need precise values for kinetic temperature to employ the $\mathrm{H}_{2} \mathrm{CO}$ "densitometer," regardless of redshift and regardless of observed lines.

For nearly all redshifts and in realistic physical conditions, the $6 \mathrm{~cm}$ line shows the strongest anti-inversion, and the antiinversion monotonically decreases with increasing line energy. Remarkably, for most scenarios, the line decrements grow from $z=0$ to $z \sim 1$, making the lines more detectable at high redshift than locally.

\section{DISCUSSION}

Our model predictions for formaldehyde excitation are consistent with observations of molecular clouds in the Galaxy, in nearby star-forming galaxies, and in a gravitational lens. Galactic dark clouds show a $6 \mathrm{~cm}$ line decrement of $T_{\mathrm{x}}-T_{\mathrm{CMB}} \simeq-0.7$ to $-0.4 \mathrm{~K}$ (Evans et al. 1975), and an LVG model applied to Galactic giant molecular clouds obtains comparable line decrements, ranging from -1 to $0 \mathrm{~K}$ (Henkel et al. 1980). The $\mathrm{H}_{2} \mathrm{CO}$ observations and radiative 
transfer modeling of star-forming galaxies in the local universe by Mangum et al. (2008) also agree with our models. The molecular cloud studied toward B0218+357 at $z=0.68$ by Zeiger \& Darling (2010) found line decrements of $\left(T_{\mathrm{x}}-\right.$ $\left.T_{\mathrm{CMB}}\right) /(1+z) \lesssim-2.1 \mathrm{~K}$ for the $6 \mathrm{~cm}$ line (for $2 \times 10^{3} \mathrm{~cm}^{-3}<$ $\left.n\left(\mathrm{H}_{2}\right)<1 \times 10^{4} \mathrm{~cm}^{-3}\right)$ and -1.5 to $-1.8 \mathrm{~K}$ for the $2 \mathrm{~cm}$ transition.

\subsection{A Formaldehyde Deep Field}

An FDF would survey the history of cosmic star formation in a fashion similar to other star formation proxies such as $\mathrm{CO}$ lines, IR/submillimeter luminosity, radio continuum, or UV light. But an FDF will differ from traditional proxies in three essential ways: (1) line detection is nearly distanceindependent and thus a survey for $\mathrm{H}_{2} \mathrm{CO}$ lines would be a dense molecular gas mass-limited survey; (2) the $\mathrm{H}_{2} \mathrm{CO}$ line strength is insensitive to gas kinetic temperature and dust temperature; and (3) $\mathrm{H}_{2} \mathrm{CO}$ lines are unaffected by dust opacity. We suspect that an FDF may be as sensitive to extended gasrich star-forming galaxies such as the "BzK" galaxies as it is to compact merger-induced starbursts such as ULIRGs and star formation-dominated submm galaxies. While the FDF concept has its shortfalls, such as a reliance on radiative transfer modeling for interpretation, it is a useful survey tool that is orthogonal to current star formation survey methods, and it overcomes the problem of obtaining redshifts for dusty galaxies identified by their dust continuum. $\mathrm{H}_{2} \mathrm{CO}$ lines select starforming gas, eliminating the uncertain active galactic nucleus dust-heating contribution to continuum star formation proxies. The strongest signals will arise from the highest molecular gas masses, which will include submm galaxies, ULIRGs, HyLIRGs, red quasar hosts, dust-obscured galaxies, and BzK galaxies.

The requirements for an FDF are: (1) beam-matched observations to the star-forming region(s) in galaxies; (2) large bandwidth, which provides a large redshift span; (3) spectral line sensitivity; and (4) either observation of more than one $\mathrm{H}_{2} \mathrm{CO}$ line or ancillary data in other parts of the EM spectrum to break a one-line redshift degeneracy. Radio interferometry meets these criteria and captures many galaxies per primary beam, but reduces the brightness temperature sensitivity. One should thus work at higher frequencies and use a more compact array, but higher frequency lines have lower optical depth and higher $T_{\mathrm{x}}$ (reduced $\Delta T_{\mathrm{Obs}}$; Figures 2 and 3). A compromise can be found, likely using the 2 or $1 \mathrm{~cm}$ lines, despite the intrinsically stronger $6 \mathrm{~cm}$ line signal.

Figure 5 shows the angular size of 2, 5, and $10 \mathrm{kpc}$ molecular regions and the Jansky Very Large Array (VLA) synthesized beam for the 1,2 , and $6 \mathrm{~cm} \mathrm{H}_{2} \mathrm{CO}$ lines for redshifts $0-10$. The $4 \mathrm{GHz}$ instantaneous frequency coverage of the $\mathrm{C}-$ band spans the remarkable redshift range $z=0.8-6.2-$ most of the epoch of galaxy evolution - because as the $2 \mathrm{~cm}$ line redshifts out of the C-band, the $1 \mathrm{~cm}$ line enters the bandpass. Even compact star-forming regions in galaxies could be beam-matched in the X-band over a significant redshift range for galaxy evolution studies: $z=0.2-0.8$ and $z=1.4$ 2.6 simultaneously. A deep observation in one of these bands would detect every galaxy above the gas mass limit in the field of view and would provide spectroscopic redshifts.

\subsection{Formaldehyde Cosmology}

Formaldehyde silhouettes of galaxies against the CMB offer a novel probe of cosmology similar to the SunyaevZel'dovich (SZ) effect in galaxy clusters. The thermal SZ

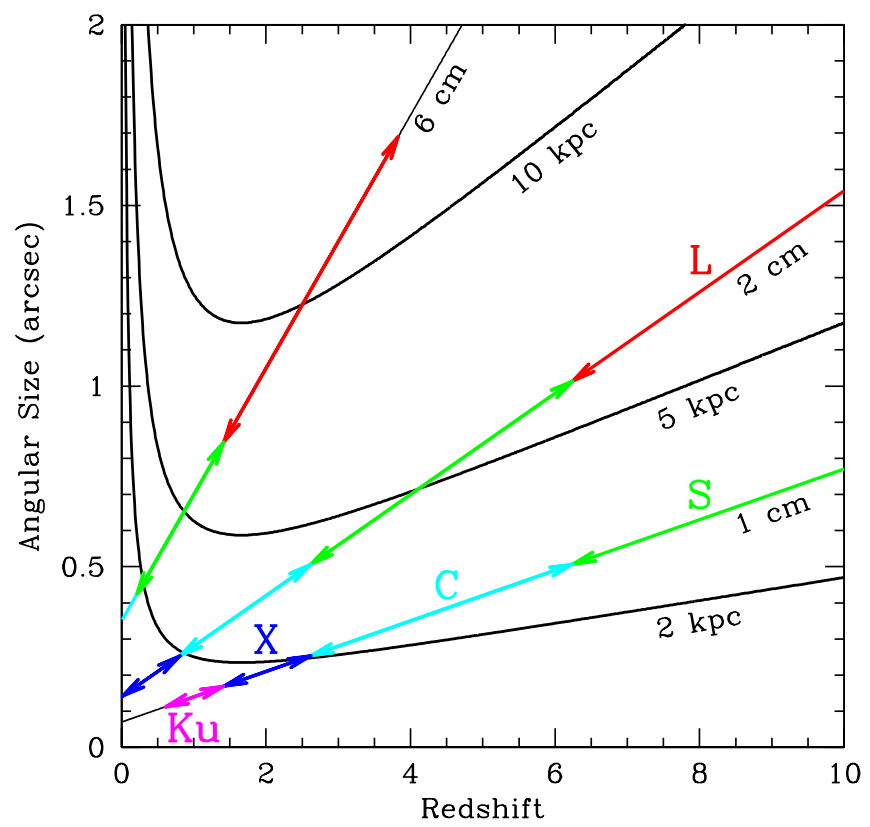

FIG. 5.- Angular sizes of fiducial 2, 5, and $10 \mathrm{kpc}$ molecular gas regions in galaxies (bold black lines) and the VLA A-array angular resolution of the 1,2 , and $6 \mathrm{~cm}$ lines of $\mathrm{H}_{2} \mathrm{CO}$ versus redshift. Galaxies can be beam-matched if the angular resolution track falls below a given physical size locus. Colors indicate the VLA wide-band receiver coverage for each line (L-band spans 1-2 GHz, S spans 2-4 GHz, C spans 4-8 GHz, X spans 8-12 GHz, and $\mathrm{Ku}$ spans $12-18 \mathrm{GHz}$ ).

effect depends on the electron density as $n_{e}$ while the X-ray emission from the cluster gas depends on $n_{e}^{2}$. A comparison of the two quantities yields a measure of the line-of-sight length scale of the cluster, $\ell$. If the proper size and line-of-sight size are equal or can be related via models, then the observed angular size of the cluster yields the angular size (or luminosity) distance:

$$
D_{A}=D_{L}(1+z)^{-2}=\frac{\ell}{\theta} \text {. }
$$

A similar measurement may be possible using $\mathrm{H}_{2} \mathrm{CO}$ absorption of the $\mathrm{CMB}$ in galaxies. $\mathrm{H}_{2} \mathrm{CO}$ line ratios indicate the local $\mathrm{H}_{2}$ density, and line depths yield the $\mathrm{H}_{2} \mathrm{CO}$ column density. Since the column density is simply the space density integrated along the line of sight, a comparison of the two quantities measures the line of sight physical size of the dense gas region of the galaxy. Assuming the line of sight and proper sizes to be equal or the galaxy has a measurable inclination, one can measure $D_{A}\left(D_{L}\right)$ from a spatially resolved spectral line map.

Neither clusters nor galaxies are spherical, but many galaxies can be observed per field of view, and in a statistical aggregate of randomly oriented galaxies, the assumption of equal size and length scales will be valid. This redshift-independent $\mathrm{H}_{2} \mathrm{CO}$ technique can employ galaxies far beyond the observed $z \sim 1$ limit on clusters, providing a unique standard ruler from the end of reionization to the formation of galaxy clusters.

While the number of galaxies with metal-rich gas at high redshift may decline compared to $z=2-3$, molecules detected in quasar hosts up to $z=6.42$ have demonstrated that high density regions in the early universe form metals and molecules quickly (e.g., Walter et al. 2003). This suggests that one may be able to identify standard rulers at very high redshift and make unprecedented cosmological measurements over a large span of the age of the universe (unlike cosmological redshift, 
$D_{L}$ and $D_{A}$ are history-integrated quantities). While the starforming regions in galaxies may show size evolution with redshift, this method does not rely on a fixed size for galaxies, only on a measurable size.

The largest possible source of uncertainty for $\mathrm{H}_{2} \mathrm{CO}$ cosmology is the $\mathrm{H}_{2} \mathrm{CO}$ abundance, which is needed to convert from $n\left(\mathrm{H}_{2}\right)$ to $n\left(\mathrm{H}_{2} \mathrm{CO}\right)$. Since $\ell \simeq N\left(\mathrm{H}_{2} \mathrm{CO}\right) / n\left(\mathrm{H}_{2} \mathrm{CO}\right)$, but $n\left(\mathrm{H}_{2}\right)=n\left(\mathrm{H}_{2} \mathrm{CO}\right) / X_{\mathrm{H}_{2} \mathrm{CO}}$ is the quantity derived from line ratios, the measured size of the molecular region in galaxies is inversely proportional to the $\mathrm{H}_{2} \mathrm{CO}$ abundance.

\subsection{Formaldehyde Contamination of H I $21 \mathrm{~cm}$ Tomography at High Redshift}

One significant concern for studies of the $\mathrm{H} \mathrm{I} 21 \mathrm{~cm}$ line before, during, and after the EoR is whether $\mathrm{H}_{2} \mathrm{CO}$ absorption of $\mathrm{CMB}$ photons may form a contaminating foreground. The main lines of interest would be those below or redshifted below $\sim 200 \mathrm{MHz}\left(z_{\mathrm{HI}}>6\right)$ : the $2_{20}-2_{21} 71.1 \mathrm{MHz}$ and $3_{21}-3_{22} 355.6 \mathrm{MHz}$ lines of para- $\mathrm{H}_{2} \mathrm{CO}$ are the best candidates (Figure 1). The ground state of the $K_{a}=2$ rotation ladder is $57.6 \mathrm{~K}$ above ground, but radiative transitions to lower para$\mathrm{H}_{2} \mathrm{CO}$ states are forbidden. As discussed in Zeiger \& Darling (2010), warm molecular gas can populate the $K_{a}=2$ states and collisions with $\mathrm{H}_{2}$ will drive these meter-wave transitions to very low excitation temperatures, of order $10-30 \mathrm{mK}$, nearly compensating for the low optical depths of these lines compared to the centimeter-wave lines. These low-frequency lines have a negligible excitation temperature compared to the $\mathrm{CMB}$ such that $\Delta T_{\mathrm{Obs}} \simeq-\left(1-e^{-\tau}\right) 2.73 \mathrm{~K} \simeq-\tau \cdot 2.73 \mathrm{~K}$, and the observed line depth is simply determined by its optical depth regardless of redshift.

Comparing $\mathrm{H}_{2} \mathrm{CO}$ meter-wave optical depths to cosmological H I $21 \mathrm{~cm}$ signals of nominal brightness temperature $20 \mathrm{mK}$ (e.g., Gnedin \& Shaver 2004), we find that the $2_{20}-2_{21}$ and $3_{21}-3_{22}$ lines are weaker than the $\mathrm{H} \mathrm{I} 21 \mathrm{~cm}$ line for $\tau<0.007$. However, our model predicts lines with $\tau \simeq 0.2$ and 0.007 , respectively. In slightly warmer $60 \mathrm{~K}$ gas, the optical depths rise to 0.4 and 0.02 , and both lines become a potentially problematic source of contamination to $\mathrm{H} \mathrm{I} 21 \mathrm{~cm}$ tomography. The $\mathrm{H} \mathrm{I}$ and $\mathrm{H}_{2} \mathrm{CO}$ signals may be separable by angular scale and spectral width, but one can imagine unresolved star formation in foreground large-scale structures near the peak of cosmic star formation at $z \simeq 1-3$ creating $\mathrm{H}_{2} \mathrm{CO}$ $355.6 \mathrm{MHz}$ line signatures that could mimic $\mathrm{H} \mathrm{I} 21 \mathrm{~cm}$ signals at $z \sim 10$.

\section{CONCLUSIONS}

Collisions with $\mathrm{H}_{2}$ cause $\mathrm{cm} \mathrm{H}_{2} \mathrm{CO}$ lines to become "antiinverted" to the point where line excitation temperatures drop below the rest-frame CMB temperature and thus create silhouettes of the dense molecular gas regions in galaxies against the uniform background. We have demonstrated using radiative transfer models that $\mathrm{cm} \mathrm{H}_{2} \mathrm{CO}$ lines observed in an FDF can provide a distance-independent extinction-free mass-limited census of the cosmic history of star formation. The detectability and interpretation of $\mathrm{H}_{2} \mathrm{CO}$ line surveys is nearly independent of redshift and gas kinetic temperature. We have also examined the possibility that $\mathrm{H}_{2} \mathrm{CO}$ lines can be used to standardize galaxies as cosmic rulers and that antiinverted meterwave $\mathrm{H}_{2} \mathrm{CO}$ lines may confuse or contaminate H I $21 \mathrm{~cm}$ tomography of the EoR. Significant new observations of $\mathrm{H}_{2} \mathrm{CO}$ at high redshift will soon be possible using wide-band interferometric facilities such as the VLA.

We are indebted to K. Eggert for writing support. We acknowledge the support of the NSF through award GSSP070015 from the NRAO and grant AST-0707713. The LUNAR consortium (http://lunar.colorado.edu) is funded by the NASA Lunar Science Institute (Cooperative Agreement NNA09DB30A).

\section{REFERENCES}

Evans, N. J. II, Zuckerman, B, Morris, G., \& Sato, T. 1975, ApJ, 196, 433

Garrison, B. J., Lester, W. A., Jr., Miller, W. H., \& Green, S. 1975, ApJ, 200, L175

Ginsburg, A., Darling, J., Battersby, C., Zeiger, B., \& Bally, J. 2011, ApJ, 736, 149

Gnedin, N. \& Shaver, P. 2004, ApJ, 608, 611

Goldreich, P. \& Kwan, J. 1974, ApJ, 189, 441

Green, S. 1991, ApJS, 76, 979

Henkel, C., Walmsley, C. M., \& Wilson, T. L. 1980, A\&A, 82, 41

Mangum, J. G., Darling, J., Menten, K. M., Henkel, C. 2008, ApJ, 637, 832

Mangum, J. G. \& Wootten, A. 1993, ApJS, 89, 123

Maret, S., \& Bergin, E. A. 2007, ApJ, 664, 956

Pagani, L., Vastel, C., Hugo, E., et al. 2009, A\&A, 494, 623
Palmer, P., Zuckerman, B., Buhl, D., \& Snyder, L. E. 1969, ApJ, 156, L147

Sobolev, V. V. 1960, Moving Envelopes of Stars (Cambridge: Harvard Univ. Press)

Townes, C. H. \& Cheung, A. C. 1969, ApJ, 157, L103

Troscompt, N., Faure, A., Maret, S., et al. 2009a, A\&A, 506, 1243

Troscompt, N., Faure, A., Wiesenfeld, L., Ceccarelli, C., \& Valiron, P. 2009b, A\&A, 493, 687

Van der Tak, F. F. S., Black, J. H., Schöier, F. L., Jansen, D. J., van

Dishoeck, E. F. 2007, A\&A, 468, 627

Walter, F., Bertoldi, F., Carilli, C., et al. 2003, Nature, 424, 406

Zeiger, B. \& Darling, J. 2010, ApJ, 709, 386 